\title{
MAGNETOHYDRODYNAMIC CHANNEL FLOW AND VARIATIONAL PRINCIPLES
}

\author{
ADNAN A. EL-HAJJ \\ Department of Mathematics \\ U.A.E. University, A1-Ain \\ United Arab Emirates \\ (Received August 26, 1986)
}

A JTRACT. This paper deals with magnetohydrodynamic channel flow problems. Attention is given to a variational principle, where the boundary conditions are incorporated via a suitable functional which is stationary at the solution of the given problem; the trial functions used for the approximate solution need not satisfy any of the given boundary conditions.

KEY WORDS AND PHRASES. Variational principle, functional, boundary conditions. 1980 AMS SUBJECT CLASSIFICATION CODE. 76 W05.

1. INTRODUCTION.

In a number of papers variational principles have been derived for the solution of linear magnetohydrodynamic channel flow of various types. Tani [1] has presented a variational principle for a problem involving the Hall effect. Smith [2] has derived a functional giving bounds on the flow rate. Sloan [3] has derived a variational principle which can be used to obtain sequences which converge to the real solution. More recently a variational principle has been derived by Barret [4] to the problem of viscous, incompressible, electrically conducting fluid flows steadily in a rectangular channel with a uniform transverse magnetic field parallel to one pair of walls, opposite pairs of walls being either insulators or perfect conductors. The equation for the nondimensional axial velocity $V$, and the nondimensional induced magnetic field $B$, are coupled and may be written as

$$
\begin{array}{ll}
\nabla^{2} V+M \frac{\partial B}{\partial x}=-1, & \\
\nabla^{2} B+M \frac{\partial V}{\partial x}=0, & |x|<1, \quad|y|<a
\end{array}
$$

where $M$ is the Hartmann number. On the boundary, $V$ is always zero and $B$ is zero if the walls are insulators and the normal derivative of $B$ is zero if the walls are perfect conductors.

Barrett and the above mentioned authors assume that the trial functions satisfy the same boundary conditions as the real field. Moreover, attempting to solve the problem of non-conducting walls, Barrett has restricted the choice of trial functions even more by reguiring that one of the governing equations be exactly satisfied. In this paper we develope a variational principle in which the given boundary conditions of the problem 
are imposed implicitly via suitable terms in the functional and hence the expansion set of functions need not satisfy any of them.

2. THE VARIATIONAL PRINCIPLE.

The variational techr ique derived in this section, applicable to a wide range of linear non-self-adjoint problems, is used to dei-ive a stationary principle for a magnetohydrodynamic channel flow. In the particular case of (1.1) and (1.2) subject to the boundary conditıons $\mathrm{V}=0$ and $\mathrm{B}=0$ a variational principle is derived by first introducing the following adjoint problem of (1.1) and (1.2):

$$
\begin{aligned}
& \nabla^{2} U-M \frac{\partial C}{\partial x}=-1 \\
& \nabla^{2} C-M \frac{\partial U}{\partial x}=0, \quad|x|<1, \quad|y|<a
\end{aligned}
$$

where $U$ and $C$ are zero on the boundary. The functional delends on four variables: $\nu, B$, $\omega$, and $T$ and is :

THEOREM 1. The solution to (1.1), (1.2), (2.1) and (2.2) renders the following functional stationary at the solution points $v=V, B=B, \omega=U$ and $T=C$ :

$$
\begin{aligned}
J(\nu, \beta, \omega, T)= & \int_{s}\{-\nabla \omega \cdot \nabla \nu-\nabla T \cdot \nabla \beta+M \omega \partial \beta / \partial x+M T \partial \nu / \partial \mathbf{x}+\nu+\omega\} d S \\
& +\int_{\partial s}\{\omega \nabla \nu \cdot \underline{\mathrm{n}}+T \nabla \beta \cdot \underline{\mathrm{n}}+\nu \nabla \omega \cdot \underline{\mathrm{n}}+\beta \nabla T \cdot \underline{\mathrm{n}}\} \mathrm{ds} \\
& -\int_{-\mathrm{a}}^{+\mathrm{a}}\left([M \nu T]_{-1}^{+1}+[M \beta \omega]_{-1}^{+1}\right) d \mathbf{y}
\end{aligned}
$$

where $\partial s$ is the boundary of the region, $\nabla \cdot \underline{n}$ denotes differentiation along the normal and $d s$ is a boundary element.

PROOF. Let $V$ and $B$ be the solution of (1.1) and (1.2); $U$ and $C$ the solution of (2.1) and $(2.2) ; \xi_{1}, \xi_{2}, \xi_{3}$ and $\xi_{4}$ arbitrary functions.

$$
\text { Define } \begin{aligned}
F_{1}(\varepsilon) & =J\left(V+c \xi_{1}, B, U, C\right) ; \quad F_{2}(\varepsilon)=J\left(V, B+\varepsilon \xi_{2}, U, C\right) ; \\
F_{3}(\varepsilon) & =J\left(V, B, U+\varepsilon \xi_{3}, C\right) ; \quad F_{4}(\varepsilon)=J\left(V, B, U, C+\varepsilon \xi_{4}\right),
\end{aligned}
$$

then the functional $J$ is stationary at $V, B, U$ and $C$ if

$$
\frac{\mathrm{dF}_{\mathrm{i}}(0)}{\mathrm{d} \varepsilon}=0, \quad i=1,2,3,4
$$

Now,

$$
\begin{aligned}
\frac{d F_{1}(0)}{d \varepsilon}= & \int_{s}\left[-\nabla U \cdot \nabla \xi_{1}+C\left(M \frac{\partial \xi_{1}}{\partial x}\right)+\xi_{1}\right] d S \\
& +\int_{\partial s}\left(U \nabla \xi_{1} \cdot \underline{n}+\xi_{1} \nabla U \cdot \underline{n}\right] d s \\
& -\int_{-a}^{+a}\left[\xi_{1} M C\right]_{-1}^{+1} d y
\end{aligned}
$$

Using Green's theorem :

$$
\int_{S}-\nabla \mathrm{U} \cdot \nabla \xi_{1} \mathrm{dS}=\int_{S} \xi_{1} \nabla^{2} \mathrm{U} d \mathcal{S}-\int_{\partial s} \xi_{1} \nabla \dot{U} \cdot \underline{\mathrm{n}} \mathrm{ds}
$$

and the fact that : 


$$
\int_{S} C\left(M \frac{\partial \xi_{1}}{\partial x}\right) d S=\int_{S}-\xi_{1} M \frac{\partial C}{\partial x} d S+\int_{+a}^{-a}\left[\xi_{1} M C\right]_{-1}^{+1} d y
$$

we get upon substituting ( $(.5)$ and $(2.6)$ in $(2.4)$ 'and recalling that on the boundary $U=0$

$$
\begin{aligned}
\frac{d F_{1}(0)}{d \varepsilon}=\int_{S} \xi_{1}\left(\nabla^{2} U-M \frac{\partial C}{\partial x}+1\right) d S & -\int_{\partial s} \xi_{1} \nabla U \cdot \underline{n} d s+\int_{-a}^{+a}\left[\xi_{1} M C\right]_{-1}^{+1} d y \\
& +\int_{\partial s} \xi_{1} \nabla U \cdot \underline{n} d s-\int_{-a}^{+a}\left[\xi_{1} M C\right]_{-1}^{+1} d y
\end{aligned}
$$

The first integral in (2.7) is equal to zero by (2.1) and it is obvious that the 1 ine Integrals cancel one another. Hence, $\mathrm{dF}_{1}(0) / \mathrm{d} \varepsilon=0$.

In much the same way, it can easily be shown that $d F_{i}(0) / d \varepsilon=0$ for $i=2,3$, 4. Hence. the functional $\mathrm{J}$ is stationary at the solutions of the given problem and its adjoint.

3. MATRIX SET-UP.

The variational principle derived in the privous section will now be used to obtain the defining equations for the approximate solution to the problem of channel flow with non-conducting walls.

We introduce appropriate basis functions - typically, a set of product orthogonal polynomials and a global expansion of the solution is made:

and

$$
v^{(N)}(x, y)=\sum_{i=1}^{N} a_{i}^{(1)} h_{i}(x, y) ; \quad \beta^{(N)}(x, y)=\sum_{i=1}^{N} a_{i}^{(2)} h_{i}(x, y)
$$

$$
\omega^{(N)}(x, y)=\sum_{i=1}^{N} b_{i}^{(1)} h_{i}(x, y) ; \quad T^{(N)}(x, y)=\sum_{i=1}^{N} b_{i}(2) h_{i}(x, y) .
$$

Inserting these expansions into (2.3) and finding the stationary value of the functional leads to the symmetric block matrix equation for the coefficients $\underline{a}^{(k)}, k=1,2$ :

$$
\left(\begin{array}{ll}
A & B \\
B & A
\end{array}\right)\left(\begin{array}{l}
\underline{a}^{(1)} \\
\underline{a}^{(2)}
\end{array}\right)=\left(\begin{array}{c}
Q \\
\underline{0}
\end{array}\right)
$$

Where for $i, j=1,2, \ldots, N$

$$
\begin{aligned}
& A=\int_{s}-\nabla h_{i} \nabla h_{j} d S+\int_{\partial s}\left(h_{i} \nabla h_{j}+h_{j} \nabla h_{i}\right) \cdot \underline{n} d s \\
& B=\int_{S} M h_{i} \partial h_{j} / \partial x d S-\int_{-a}^{+a}\left[M h_{i} h_{j}\right]_{-1}^{+1} d y \\
& \underline{Q}=\int_{S}-h_{i} d S \\
& \underline{0}=\text { zero vector of appropriate length. }
\end{aligned}
$$

An important feature of our technique is that the basis functions $h_{i}(x, y)$ are not required to satisfy any of the boundary conditions of the problem; these conditions are imposed implicitly by the functional, and are satisfied exactly only at the solution point. We only require, for stability reasons ( see Mikhiln [5]), that the set of basis functions be a set of product orthogonal polynomials. 
To solve (3.2) a numerical technique which reduces the operation count and leads to economic use of storage will be used in a forthcoming paper together with the present varıatıonal formalısm to produce numerical results for the magnetohydrodynamic channel flow problem.

\section{PEFERENCES}

1. TANI, I. Steady Flow of Conducting Fluids in Channels under Transverse Magnetic Fields, with Consideration of Hall Effect, J.Aero. Sci.29 (1962), 297-304.

2. SMITH, P. Some Extremum Principles tor Pipe Flow in Magnetohydrodynamics, Z. angew. Math. Phys. 23 (1972), 753-764.

3. SLOAN, D.M. Extremum Principles for Magnetohydrodynamic Channel Flow, Z. angew. Math. Phys. 24 (1973), 689-698.

4. BARRETT, K.E. Mınimax Principle for Magnetohydrodynamic Channel Flow, ZAMP 27 (1976), 613-619.

5. MIKHILN, S.G. The Numerical Performance of Variational Methods, Noordhoff, Amsterdam, 1971 . 


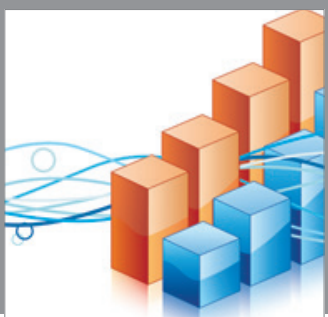

Advances in

Operations Research

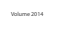

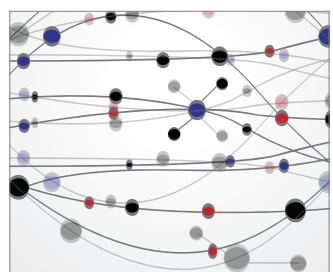

\section{The Scientific} World Journal
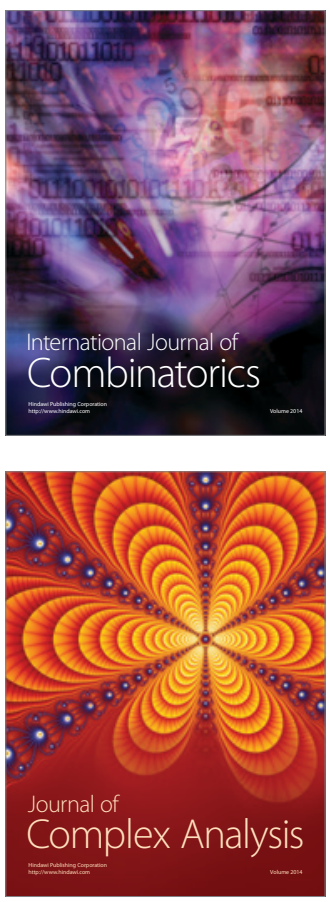

International Journal of

Mathematics and

Mathematical

Sciences
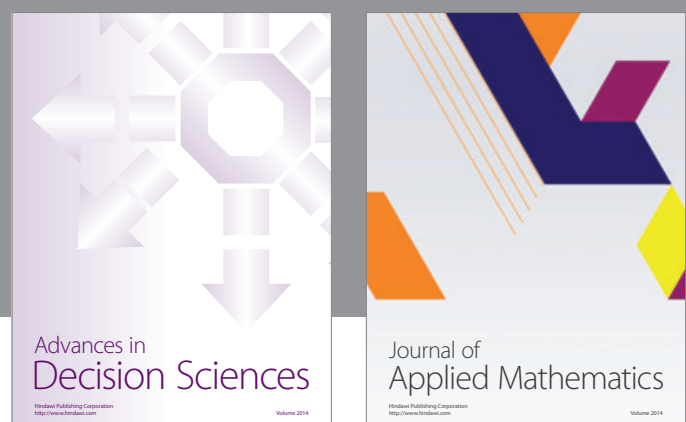

Journal of

Applied Mathematics
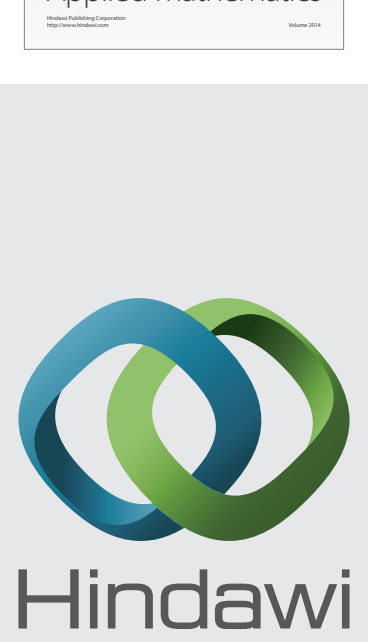

Submit your manuscripts at http://www.hindawi.com
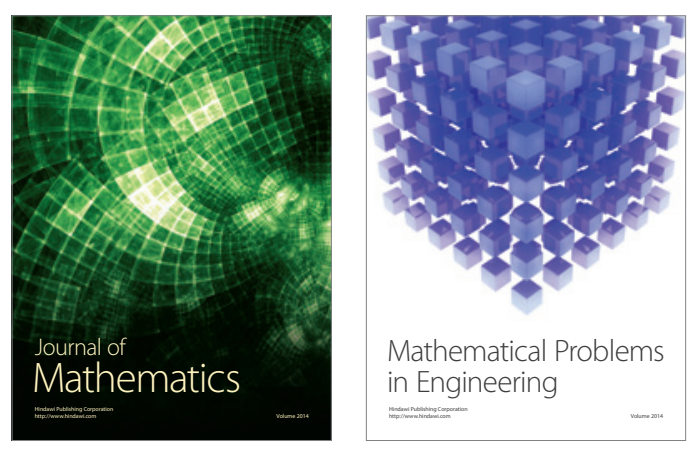

Mathematical Problems in Engineering
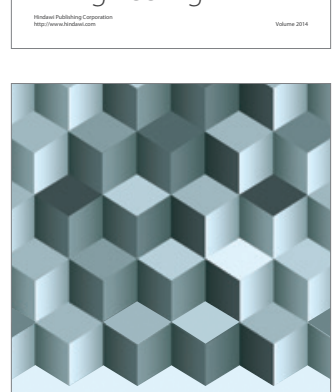

Journal of

Function Spaces
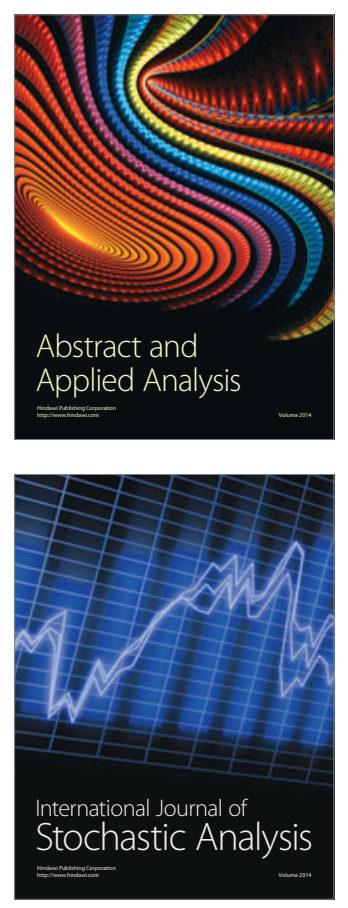

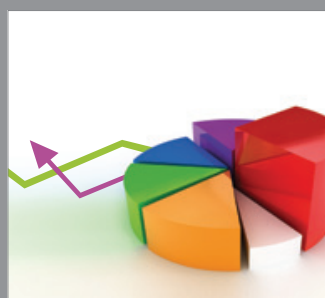

ournal of

Probability and Statistics

Promensencen
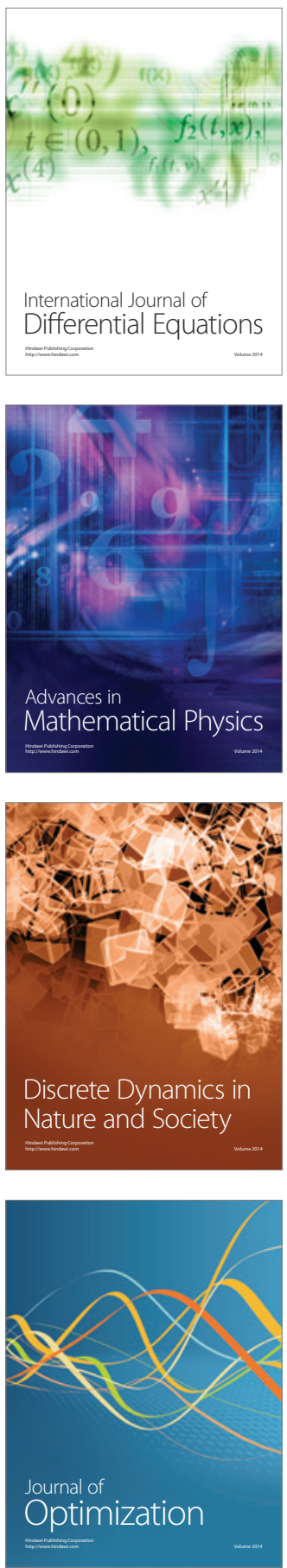